\title{
¿Y en qué lugar se enamoró de ti? Inmigración internacional y endogamia conyugal ${ }^{1}$
}

\section{Clara Cortina}

Consejo Superior de Investigaciones Científicas. Centro de Ciencias Humanas y Sociales clara.cortina@cchs.csic.es

\section{Albert Esteve}

Centre d'Estudis Demogràfics

aesteve@ced.uab.es

\section{Resumen}

Usando los datos de la Encuesta Nacional de Inmigrantes de 2007, este artículo tiene dos objetivos: a) examinar los niveles de endogamia de la población inmigrada en España que formó pareja tras la migración, y $b$ ) analizar la variación de los niveles de endogamia según la situación de pareja y la edad en el momento de llegar. Los resultados muestran diferencias importantes en función del colectivo de inmigrantes. Los africanos son los más endógamos, seguidos de los hombres de Europa Oriental y América Latina. Las mujeres de estos orígenes, en cambio, se unen en más de un 50\% con hombres españoles. Por su parte, aproximadamente cuatro de cada cinco inmigrantes de la Europa Occidental lo hacen con un cónyuge español. En función del criterio para seleccionar a los inmigrantes, los niveles de endogamia varían. Los datos analizados sugieren que la edad de llegada puede utilizarse como una variable de filtro cuando no es posible conocer la situación conyugal de los inmigrantes en el momento de llegar a España. A edades más jóvenes, menor es la posibilidad de haber formado pareja antes de la migración.

Palabras clave: migración internacional; matrimonio; familia.

\section{Abstract. The endogamy of international migrants in Spain}

The objective of this paper is twofold. First, it examines endogamy rates among international migrants who were not in union at the time of arrival to Spain. Second, it compares endogamy levels for different ages at arrival and partnership status at arrival. Data come

1. Este artículo se ha realizado en el marco del proyecto del Plan Nacional del Ministerio de Ciencia e Innovación El impacto de la población extranjera en la formación de la pareja en España (SEJ2007-60014), y del proyecto del Instituto de la Mujer Mujeres en tránsito y transformación de la identidad de género en los procesos migratorios: un enfoque interdisciplinario (Ref.6/10). 
from the National Immigrant Survey (2007). Results show that endogamy rates vary by country of birth. Immigrants from Africa are the most endogamous, followed by East European and Latin-American men. Conversely, more than $50 \%$ of women from these two regions are married or cohabit with a Spanish man. Almost 4 out of 5 immigrants from the EU15 are in union with a Spanish partner. The analysis show that endogamy rates depend on the specification of unions. Age at arrival can be used as a selection criterion when the partner status at the time of arrival is not available. At younger ages, migrants are less likely to be in union.

Key words: international migration; marriage; family.
Sumario
1. Introducción
5. Niveles de endogamia de la población
2. La endogamia conyugal inmigrada. Situación de pareja y edad de los inmigrantes internacionales en el momento de migrar
3. El estudio de la endogamia conyugal 6. En resumen y conclusión de los inmigrantes en España Referencias bibliográficas

4. Datos y métodos

\section{Introducción}

La intensificación de los flujos de inmigración internacional hacia España ha conllevado un aumento de la población extranjera y, con ello, un interés creciente por la integración de los inmigrantes en distintos ámbitos de la sociedad. En el ámbito de la formación de la pareja, las uniones mixtas entre nativos e inmigrantes son un indicador clásico de integración. Desde perspectivas distintas, teorías como las de la asimilación, la asimilación segmentada o el intercambio colectivo destacan la importancia social de este tipo de uniones. Numerosos estudios identifican los factores individuales y estructurales asociados con la formación de parejas mixtas por parte de los inmigrantes y de minorías étnicas y raciales. A nivel individual, el capital humano, los años de residencia en el país, la posición social y el dominio de la lengua autóctona suelen estar correlacionados con una mayor propensión a unirse fuera del grupo. A nivel estructural, se consideran aspectos como el tamaño del grupo, el grado de segregación territorial o la relación de masculinidad. El grueso de la literatura sobre este tema procede de sociedades con una larga y dilatada experiencia migratoria, como, por ejemplo: Estados Unidos, Reino Unido, Francia, Holanda y Suecia.

Los trabajos realizados en España sobre dicho tema se han hecho eco de esta literatura. Los primeros estudios cuantitativos se basaron en datos del censo de 2001 (en un contexto de poca inmigración) y del registro de matrimonios del Movimiento Natural de la Población (MNP). La novedad del fenómeno migratorio en España obligaba a tratar con precaución los resultados obtenidos 
y a no implementar directamente las hipótesis generadas en otros contextos. Todavía hoy, la experiencia inmigratoria de España es breve. El peso de la primera generación de inmigrantes (entendida como aquella que protagonizó la inmigración) es mayoritario en relación con la segunda y ulterior generación (los descendientes de los inmigrantes nacidos en España). Extraer conclusiones de las pautas de emparejamiento de la población inmigrada es arriesgado, porque se desconoce si el inmigrante formó pareja antes o después de la migración. Sin datos que permitan conocer la situación de pareja en el momento de la migración, existe el riesgo de sobreestimar a los matrimonios endógamos (dentro del grupo), dado que la mayoría de los inmigrantes que tienen pareja antes de la migración están unidos con alguien de su mismo origen. Hasta la publicación de la Encuesta Nacional de Inmigrantes de 2007, no fue posible conocer la situación conyugal ni el origen del cónyuge en el momento de llegar a España. El Censo de Población y la Encuesta de Población Activa no permiten distinguir quien llegó unido de origen de quien se unió en destino. Por su parte, el MNP, que incluye los matrimonios celebrados en España, no capta ni las uniones consensuales ni los matrimonios entre extranjeros que se forman tras la migración en los países de origen.

Este trabajo tiene dos objetivos principales. En primer lugar, con datos de la Encuesta Nacional de Inmigrantes de 2007, se estiman los niveles de endogamia de las uniones que ha formado la población inmigrada residente en España en 2007 y que no tenía pareja en el momento de la llegada, distinguiendo entre hombres y mujeres y combinando matrimonios y uniones consensuales. En segundo lugar, se analiza la variación de los niveles de endogamia según la situación de pareja y la edad en el momento de la llegada. El propósito es examinar la validez de la edad de llegada como variable de filtro de los inmigrantes que llegaron sin pareja cuando no se conoce la situación de pareja a la llegada.

\section{La endogamia conyugal de los inmigrantes internacionales}

El estudio de la formación de la pareja entre los inmigrantes internacionales se justifica por varias razones. Primero, informa de las posibilidades que tienen los migrantes de llevar una vida en pareja en el país de destino y de formar una familia (González Ferrer, 2008). Si la pareja existe antes de la migración, informa de las posibilidades de migrar conjuntamente o, cuando uno de los miembros ha migrado primero, de reagrupar el cónyuge ausente (Lázaro González, 2002). Todo ello tiene implicaciones para la integración y el asentamiento de los migrantes en la sociedad de destino (González Ferrer, 2006). Segundo, las pautas de emparejamiento de los extranjeros han sido leídas en clave de distancia social entre grupos (Price y Zubrzycki, 1962; Pagnini y Morgan, 1990; Kalmijn, 1998). Un elevado número de parejas mixtas es utilizado como indicador de asimilación de tipo estructural (Gordon, 1964). Desde una óptica microsocial, las parejas mixtas son consideradas como células de hibridación cultural en un mundo globalizado (Filhon y Varro, 2005; Collet y Philippe, 2008). La estabilidad y el conflicto asociado a estas parejas, la negociación 
entre identidades culturales distintas en las decisiones del día o la educación de los hijos ha concitado el interés de antropólogos y sociólogos (Rodríguez Marcos, 2006). También la inmigración internacional introduce una nueva dimensión en el mercado matrimonial que altera el comportamiento nupcial de la población autóctona.

Para explicar las diferencias en las propensiones a formar matrimonios dentro y fuera del grupo, la literatura ha distinguido fundamentalmente entre factores individuales y factores estructurales o de mercado (Kalmijn, 1998). El papel del mercado matrimonial es clave para explicar la probabilidad de hombres y mujeres de unirse fuera del grupo cuando hay desequilibrios por sexo dentro del mismo. Es de esperar que los hombres y las mujeres que presenten un mayor déficit respecto al sexo opuesto de su mismo origen, sean las personas que muestren mayores niveles de exogamia. En aquellos colectivos en los que los hombres son deficitarios, las mujeres suelen contraer más nupcias con hombres de otra nacionalidad residente en España o bien importan consortes de sus países respectivos, y viceversa para las mujeres. En cualquier caso, la delimitación del mercado matrimonial de los inmigrantes es tarea difícil. Definir los candidatos en función de su origen y contabilizarlos en destino es sólo un intento parcial de dimensionar el mercado real de esposos potenciales, que a menudo se extiende también hasta el país de origen. Las dinámicas transnacionales se expresan también en la esfera de las relaciones familiares y de parentesco e influyen en momentos clave, como el de la elección de la pareja (Lievens, 1999).

Más allá de la influencia de los determinantes estructurales del número de efectivos, teorías como la de la asimilación (Alba y Nee, 1997), la de la asimilación segmentada (Portes y Zhou, 1993) o la del intercambio social (Merton, 1941) coinciden en significar la importancia de las uniones mixtas. Las teorías de la asimilación asumen que los grupos de inmigrantes generan estrategias propias basadas en su caracterización sociodemográfica y su proceso migratorio. Por su lado, la teoría del intercambio social se basa en el capital humano y supone que el bajo valor del origen inmigrante en el mercado laboral puede ser contrarrestado por un elevado capital humano. De acuerdo con esto, la endogamia debería ser menor entre los inmigrados y las inmigradas con un mayor nivel de estudios.

Estas teorías surgieron a raíz de trabajos sobre parejas mixtas (intermarriage) producidos en sociedades con mayor experiencia inmigratoria, básicamente del mundo anglosajón. Sin embargo, es discutible la conveniencia de estos enfoques para investigar el caso español, dado el carácter reciente del fenómeno inmigratorio. El estudio de la formación de la pareja entre las primeras generaciones exige un enfoque más dinámico que incorpore elementos del ciclo de vida del inmigrante antes, durante y después de la migración. Conocer, por ejemplo, si el inmigrante o la inmigrante tenía pareja antes de la migración, si ambos cónyuges migraron conjuntamente, si existió un proceso de reagrupación posterior de uno de los cónyuges, entre otras trayectorias, resulta clave para entender la dinámica familiar de estos colectivos. Probablemente, ha sido en el ámbito de la fecundidad de las mujeres inmigradas donde encontramos 
más ejemplos de esta interacción (Devolder y Treviño, 2007; Roig y Castro Martín, 2007; Sobotka, 2008). En relación con la interacción entre migración y formación de la pareja, existe un vacío importante, no sólo en España, sino también en otros países.

\section{El estudio de la endogamia conyugal de los inmigrantes en España}

La población extranjera ha tenido un impacto notable en la formación de la pareja en España. El número de matrimonios mixtos celebrados en España ha crecido de 9.267 a 28.061 entre 1998 y 2008. En 2008, estos matrimonios representaban el 14,5\% del total de matrimonios celebrados en España en este último año y representan aproximadamente el $75 \%$ de los matrimonios con al menos un extranjero. Además, existe una notable asimetría de género en el impacto que la población extranjera ha tenido en la formación de la pareja para la población española (Cortina, Esteve y Domingo, 2006; Cortina, García y Esteve, 2009). Por ejemplo, desde 1999, son más numerosos los matrimonios de hombre español con mujer extranjera (especialmente latinoamericanas) que la combinación inversa. Los primeros resultados extraídos del MNP y la Encuesta de Población Activa también muestran diferencias relevantes entre sexos: las mujeres españolas en uniones mixtas tienen un comportamiento parecido (edad que tienen cuando se casan, diferencia de edad) al de las mujeres que se unen con españoles. No ocurre lo mismo con los hombres españoles que se unen con extranjeras. La edad que tienen cuando se casan es más tardía y la diferencia de años entre cónyuges es mayor comparada con los matrimonios entre dos españoles.

En el terreno metodológico, se han realizado importantes avances (Cortina, Esteve y Cabré, 2009; Esteve y Bueno, 2010). Las cuestiones conceptuales y metodológicas son una preocupación central de este tipo de investigaciones. Con las fuentes disponibles en España (Censo de Población y Encuesta de Población Activa), los inmigrantes pueden identificarse según dos criterios: el país de nacimiento y/o el país de nacionalidad. Los resultados muestran que la utilización de uno u otro criterio tiene implicaciones para el análisis y se minimiza la medición de la endogamia cuando se utiliza la nacionalidad (algunas parejas mixtas por nacionalidad pueden esconder una unión entre dos cónyuges del mismo origen porque uno de ellos ya haya adquirido la nacionalidad española). Este efecto varía entre colectivos en función de su acceso a la nacionalidad española (Esteve y Bueno, 2010).

Con las fuentes estadísticas disponibles hasta la publicación de la Encuesta Nacional de Inmigrantes, no es posible clasificar las uniones según el momento y el lugar de celebración. Las investigaciones realizadas con el Censo y la EPA han evidenciado discrepancias entre las mismas atribuibles básicamente al universo de uniones que registran. El MNP informa exclusivamente de las parejas unidas en matrimonio en territorio español y, por tanto, ofrece una visión parcial de las parejas de extranjeros y de su composición. El MNP ofrece una visión del nivel de interacción matrimonial entre extranjeros y españoles que no se corresponde con ninguna otra fuente (Censo, Encuesta de Población 
Activa, información de las parejas con hijos de las estadísticas de nacimientos). Los niveles de endogamia de los inmigrantes en el MNP son notablemente inferiores a los que se observan cuando se considera el conjunto de parejas (Esteve y Bueno, 2010). Los datos del resto de fuentes coinciden en afirmar el predominio de la endogamia en más de un $80 \%$ de las parejas (Cortina, Esteve y Domingo, 2008; Cortina, Esteve y Cabré, 2009). Existen, sin embargo, diferencias entre colectivos, así como entre hombres y mujeres dentro de un mismo colectivo, e incluso entre personas de un mismo sexo y un mismo colectivo. En consonancia con investigaciones de otros países, hay factores de tipo estructural e individual que favorecen una mayor exogamia (Blau et al., 1982; Massey, 1985; McCaa, 1993; Blackwell, 1998; Qian, 1998; Jacobs y Labov, 2002). Sin embargo, aún controlando por estos factores, hay colectivos más propensos a la exogamia que otros o, por ejemplo, con una mayor presencia de uniones consensuales (Cortina, Esteve y Domingo, 2008; Esteve y Jiménez, 2010). Este hecho sugiere la existencia de factores de tipo cultural que expliquen las diferencias entre colectivos (Lievens, 1999).

La escasez de información biográfica acerca de las parejas y los individuos ha sido, sin duda alguna, la principal limitación de los trabajos citados. En ocasiones, la escasez de datos ha sido compensada por estudios de corte cualitativo (Rodríguez, 2004, 2006; Bledsoe et al., 2007). Los resultados obtenidos han puesto en entredicho la capacidad de las fuentes estadísticas para ofrecer una visión completa y dinámica de las uniones de extranjeros en España. Los principales problemas son: se desconoce si las parejas se formaron antes o después de la migración; no se sabe si la nacionalidad se obtuvo antes o después del matrimonio; tampoco se sabe si los miembros de la pareja migraron al mismo tiempo o si uno de los miembros fue reagrupado con posterioridad; se desconoce dónde se conocieron los cónyuges; se ignora si los inmigrantes solteros y en situación regular «importan» a sus parejas de sus países de origen respectivos. Tampoco es posible identificar las segundas generaciones. No hay información de las características de los hijos de inmigrantes nacidos en España y de nacionalidad española, una generación clave para examinar la integración de estos colectivos en nuestra sociedad.

Afortunadamente, el Instituto Nacional de Estadística publicó, en julio de 2008, los microdatos de la Encuesta Nacional de Inmigrantes (ENI) realizada en 2007. Una encuesta que está teniendo un papel destacado en los estudios sobre inmigración en España (Reher y Requena, 2009). Esta fuente permite contrastar la visión de las pautas de emparejamiento de los extranjeros ofrecida por las fuentes utilizadas hasta el momento (Censo, EPA y MNP) y evaluar la funcionalidad de la edad a la migración como proxy de la situación de pareja a la llegada.

\section{Datos y métodos}

\subsection{La Encuesta Nacional de Inmigrantes}

Los datos utilizados en esta investigación proceden de la Encuesta Nacional de Inmigrantes (ENI), publicada por el Instituto Nacional de Estadística y reali- 
zada entre noviembre de 2006 y febrero de 2007. Se trata de una encuesta con información retrospectiva basada en una muestra de 15.465 personas nacidas en el extranjero de dieciséis años o más en el momento de la encuesta que llevan residiendo en España al menos un año o, no siendo así, que tiene intención de residir en el país al menos un año. Los nacidos en el extranjero con nacionalidad española de nacimiento que llegaron a España antes de cumplir los dos años de edad quedaron excluidos del ámbito poblacional de la encuesta. Para los individuos entrevistados, la encuesta proporciona información de sus características sociodemográficas, experiencia migratoria, condiciones en el país de partida, llegada a España, historia laboral y residencial, relación con el país de nacimiento, participación social y situación documental. Adicionalmente, se tiene información sobre los miembros con los que comparte vivienda el entrevistado. Esta información se limita al país y a la fecha de nacimiento, al sexo, al año de llegada a España, al país o países de nacionalidad, a la nacionalidad española y al año de adquisición, si corresponde. Cuando el esposo o la esposa no convive con el entrevistado, éste último proporciona la información de su pareja. Se trata de las mismas variables que se conocen para los miembros de la vivienda más el lugar de residencia en el momento de la encuesta.

Siguiendo las recomendaciones del Instituto Nacional de Estadística, los resultados se presentan a nivel nacional y para cuatro grupos de inmigrantes en función de la región de origen: África, América Latina, Europa de los Quince y Europa Oriental, más una categoría residual de «Otros» ${ }^{2}$. La densidad de las muestras no permite una desagregación más detallada por país de nacimiento, a excepción de Marruecos, Ecuador y Rumanía. De hecho, estos son los colectivos que tienen un peso mayor dentro de los inmigrantes procedentes de África, América Latina y Europa Oriental. Tener que renunciar a trabajar con una desagregación mayor impide, por ejemplo, analizar a los asiáticos y supone romper la serie de comparabilidad con análisis precedentes realizados con el censo de 2001 (Cortina, Esteve y Domingo, 2008). La identificación de los inmigrantes se realiza a partir de la variable pais de nacimiento. Los inmigrantes emparejados con un cónyuge nacido en el mismo país o con un cónyuge que tenga la nacionalidad de su país de nacimiento son considerados endógamos.

\subsection{Reconstrucción de las trayectorias de pareja}

Las trayectorias migratorias y de pareja se construyen a partir de la situación de pareja en tres momentos del tiempo: antes de la migración, en el momento de la migración y en el momento de la encuesta. Antes, sin embargo, es preciso aclarar algunos aspectos. En primer lugar, estas trayectorias no tienen duraciones, porque los eventos relacionados con la formación de la pareja no están

2. Se restringe la categoría de Unión Europea a los quince primeros países y se crea la categoría de Europa Oriental, porque Rumanía y Bulgaria, de donde proceden muchos inmigrantes, no se incorporaron a la UE hasta 2007, fecha de realización de la ENI. 
fechados, a excepción del año del último matrimonio. En segundo lugar, se desconoce si han existido cambios de estado en la situación de pareja entre el momento de la migración y el momento de la encuesta, ya que la fuente no proporciona información sobre la historia matrimonial y de parejas estables de los inmigrantes. Así, por ejemplo, si un inmigrante llegó con su pareja a España y convive en el momento de la encuesta con una pareja extranjera que llegó en el mismo año, se asume que se trata de la misma persona. En tercer lugar, se pueden identificar matrimonios y uniones consensuales, tanto en origen como en destino, si bien sólo se conoce la fecha del matrimonio vigente, y no la del inicio de la unión consensual vigente. Esto dificulta la realización de modelos de duración que tengan en cuenta el tiempo entre la llegada y la formación de la pareja entre los cohabitantes.

Las trayectorias satisfacen sobradamente los objetivos de esta investigación. En primer lugar, se clasifica a los individuos según su situación de pareja en el momento de migrar a través de la pregunta: «¿Dónde vivía su pareja en el momento de llegar a España?». Las personas que respondieron «No tiene pareja» a esta pregunta son consideradas sin pareja. Esta categoría incluye a solteros, separados, divorciados y viudos que declararon no tener pareja en el momento de llegar a España, pero no se pueden diferenciar por el estado civil, porque se desconoce este dato antes de la migración. Las personas que declararon tener pareja en el mismo hogar son consideradas con pareja, cónyuge presente. Y las personas que tenían a su pareja fuera del país se consideran con pareja, cónyuge ausente $^{3}$. En segundo lugar, se clasifica a los individuos según la situación de pareja actual. Esta viene determinada por la presencia o la ausencia de un cónyuge entre los miembros de la vivienda, la información de acuerdo con las relaciones de parentesco entre la persona entrevistada y el resto de miembros del hogar. Los que no tienen cónyuge en la vivienda, pero sabemos que está ausente gracias a una pregunta que se realiza directamente al entrevistado, son considerados con pareja, cónyuge ausente. El resto son sin pareja.

Combinando estos dos momentos, se obtienen las distintas trayectorias, en su versión más simplificada:

1) Individuo que no tenía pareja en el momento de llegar y sigue sin tener pareja en el momento de la encuesta.

2) Individuo que no tenía pareja en el momento de llegar y tiene pareja en el momento de la encuesta. Son personas que se han emparejado después de haber llegado a España, tanto aquí como en su país de origen, en cuyo caso estaríamos hablando de aquellos casos que, en la literatura, suelen denominarse «importadores» del cónyuge ${ }^{4}$.

3. En una proporción reducida de casos $(3,9 \%)$, la pareja del inmigrante no residía ni en el mismo hogar ni fuera del país, sino en el mismo país pero en distinta vivienda, la gran mayoría, en el mismo municipio (64.3\%). Hemos asignado estos casos a la categoría con pareja, cónyuge presente, sin perjuicio para los resultados finales.

4. No se distinguen aquí unos de otros, pero es posible hacerlo con la información que proporciona la encuesta (ver González Ferrer, 2011). 
Tabla 1. Distribución de los inmigrantes según su situación de pareja en su llegada a España, por sexo y lugar de nacimiento

\begin{tabular}{|c|c|c|c|c|c|c|}
\hline & $\begin{array}{c}\text { Sin pareja antes } \\
\text { de migrar y en la } \\
\text { encuesta }\end{array}$ & $\begin{array}{l}\text { Han formado } \\
\text { pareja tras } \\
\text { migrar }\end{array}$ & $\begin{array}{l}\text { Migraron } \\
\text { teniendo } \\
\text { pareja }\end{array}$ & Otros & $\%$ & Total \\
\hline \multicolumn{7}{|l|}{ Hombres } \\
\hline Marruecos & 33,7 & 31,7 & 25,4 & 9,1 & 100 & 1.160 \\
\hline Ecuador & 24,1 & 20,3 & 47,0 & 8,6 & 100 & 602 \\
\hline Rumanía & 29,2 & 17,1 & 51,0 & 2,7 & 100 & 778 \\
\hline \multicolumn{7}{|l|}{ Resto de } \\
\hline Latinoamérica & 30,0 & 23,5 & 41,1 & 5,4 & 100 & 2.145 \\
\hline Resto de África & 38,8 & 20,8 & 32,9 & 7,6 & 100 & 554 \\
\hline Asia & 37,4 & 22,0 & 30,4 & 10,2 & 100 & 431 \\
\hline Unión Europea & 24,5 & 32,7 & 38,6 & 4,2 & 100 & 1.601 \\
\hline Resto del mundo & 25,3 & 24,6 & 42,6 & 7,5 & 100 & 695 \\
\hline Total & 29,5 & 25,5 & 38,7 & 6,3 & 100 & 7.966 \\
\hline \multicolumn{7}{|l|}{ Mujeres } \\
\hline Marruecos & 28,6 & 27,8 & 35,4 & 8,2 & 100 & 658 \\
\hline Ecuador & 30,8 & 21,9 & 38,2 & 9,0 & 100 & 651 \\
\hline Rumanía & 23,0 & 20,9 & 48,4 & 7,7 & 100 & 675 \\
\hline \multicolumn{7}{|l|}{ Resto de } \\
\hline Latinoamérica & 32,3 & 25,9 & 33,5 & 8,3 & 100 & 2.626 \\
\hline Resto de África & 28,6 & 26,7 & 37,6 & 7,1 & 100 & 211 \\
\hline Asia & 22,5 & 29,2 & 34,0 & 14,2 & 100 & 253 \\
\hline Unión Europea & 23,2 & 34,7 & 34,1 & 8,0 & 100 & 1.514 \\
\hline Resto del mundo & 24,3 & 27,0 & 38,5 & 10,2 & 100 & 699 \\
\hline Total & 27,8 & 27,3 & 36,2 & 8,6 & 100 & 7.287 \\
\hline
\end{tabular}

Fuente: Encuesta Nacional de Inmigrantes, INE, elaboración propia.

Nota: casos ponderados.

3) Individuo que tenía pareja al migrar. Se incluyen aquí a los que migraron con ella, a los que fueron reagrupados por sus cónyuges, a los que reagruparon a sus cónyuges y a los que, teniendo pareja, migraron solos y siguen sin residir con su pareja en España ${ }^{5}$. Por último, se considera una categoría adicional para aquellas situaciones que se escapan de la clasificación propuesta, en parte por las limitaciones de los datos.

La tabla 1 presenta la distribución de los hombres y las mujeres inmigrantes de cada país o región de origen según sea su trayectoria combinada de migración y formación de pareja. Los individuos cuyas trayectorias se representan en esta tabla tienen dieciséis años o más en el momento de realizar la encuesta,

5. Para distinguir estas situaciones (si la persona migró junto a su pareja o en solitario y, en este último caso, diferenciamos entre si migró primero o en segundo lugar), se pueden utilizar las respuestas a otras dos preguntas de la encuesta: «¿En qué año llegó a España?», pregunta realizada a ambos cónyuges, y «¿Con quien se trasladó a este país?». 
pero pudieron haber llegado a cualquier edad. Esto explica que, en términos generales, la mayoría de los inmigrantes llegaron a España sin pareja, aproximadamente el 55\%, tanto en el caso de hombres como de mujeres, si sumamos los que siguen sin tener pareja y los que ya están unidos. Una cuarta parte de los hombres $(25,5 \%)$ y de las mujeres $(27,3 \%)$ han llegado a España sin tener pareja, pero estaban unidos en el momento de la encuesta. Esto equivale a prácticamente la mitad de los que no tenían pareja en el momento de llegar. El resto de trayectorias corresponden a los que tenían pareja en el momento de llegar.

\subsection{El estado conyugal de los inmigrantes en el momento de la migración}

El gráfico 1 muestra la proporción de inmigrantes internacionales que declararon no tener pareja en el momento de la migración por edad de llegada ${ }^{6}$. El gráfico distingue entre hombres y mujeres y por origen de los mismos. Como se ha visto en el apartado anterior, el hecho de no tener pareja en el momento de la llegada no significa que la persona sea soltera o no haya tenido una relación previa a la migración, simplemente indica que el inmigrante no tenía pareja en el momento de migrar. La proporción de inmigrantes sin pareja decrece con la edad de llegada. Lógicamente, cuando más elevada es la edad de llegada, mayor es la probabilidad de tener pareja en dicho momento. Las diferencias en el calendario de entrada a la unión entre hombres y mujeres y entre regiones del mundo explican buena parte de las diferencias entre las curvas representadas. La proporción de hombres que llega sin tener pareja es superior al 50\% hasta los veintiséis años para latinoamericanos y de la Europa Oriental, treinta años para los africanos y treinta y dos para los europeos comunitarios. Entre las mujeres, esta cifra se alcanza entre los diecinueve y los veintiún años para africanas y latinoamericanas, a los veinticuatro para las mujeres procedentes de la Europa Oriental, y entre los veintinueve y treinta años entre las mujeres de la Europa de los Quince. En el caso de africanas y latinoamericanas, se observa un cierto repunte de la proporción de mujeres sin pareja a partir de los treinta años (un poco antes para las africanas). Esto puede ser el reflejo de migración femenina independiente, a menudo protagonizada por mujeres separadas o divorciadas.

\subsection{Otros aspectos metodológicos}

El análisis descriptivo se completa con tres regresiones logísticas que comparan los niveles de endogamia matrimonial controlando las características de los grupos. La Encuesta Nacional de Inmigrantes informa del año de

6. Las proporciones representadas han sido ajustadas utilizando una función polinómica para suavizar las oscilaciones en las proporciones observadas debidas al reducido número de casos. Las funciones ajustan con un $\mathrm{R}^{2}$ superior a 0,7 en todos los casos, tanto para hombres como para mujeres. 
Gráfico 1. Proporciones ajustadas de migrantes que no tenían pareja al migrar, por edad a la llegada, sexo y origen

\section{Hombres}

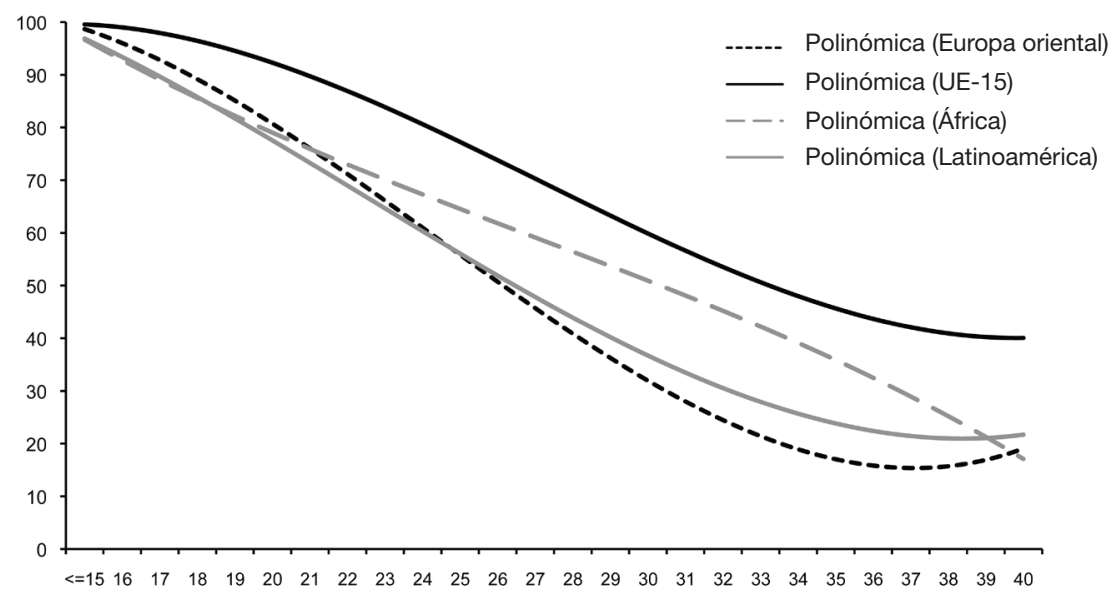

Mujeres

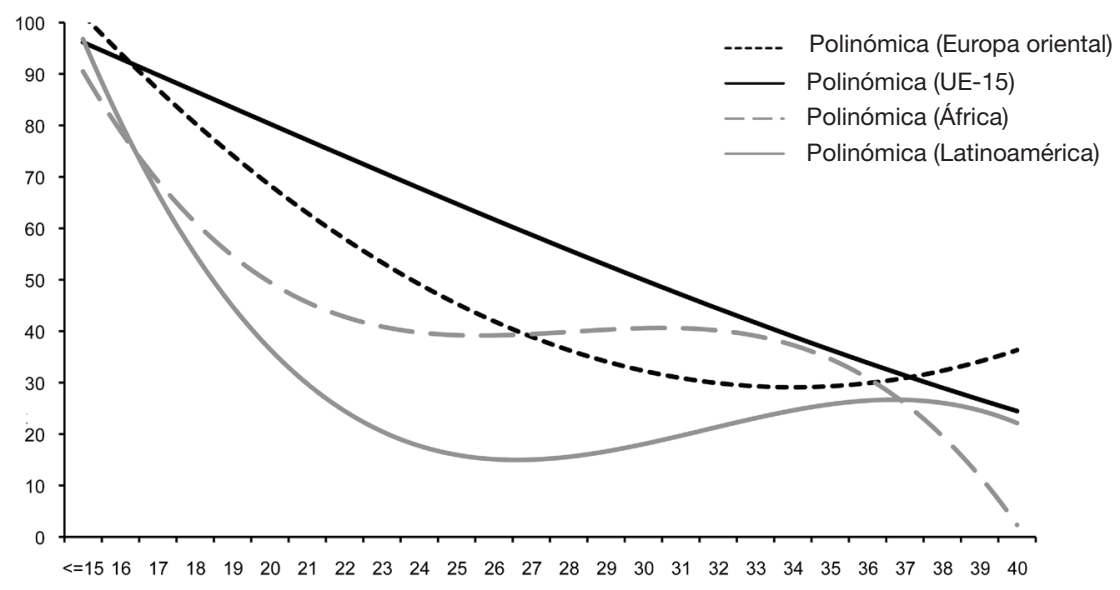

Fuente: Encuesta Nacional de Inmigrantes, INE, elaboración propia. Nota: casos ponderados. 
matrimonio, pero no del año de entrada en unión. Por esta razón, si se quieren conservar las uniones consensuales en el análisis, hay que prescindir de la variable duración.

Concretamente, se realizan tres modelos para hombres y tres para mujeres y se introduce la variable región de origen como principal variable de interés junto con algunas variables de control. El primero de ellos está basado en la población inmigrante que no tenía pareja en el momento de la llegada. El segundo incluye a todos los inmigrantes, pero añade una variable dicotómica que distingue a los que tenían pareja de los que no la tenían. El tercer modelo incluye a todos los inmigrantes clasificados por edad de llegada en seis grupos $(<15,15-19,20-24,25-29,30-34,35+)$. A diferencia de lo que ocurre en el primer modelo, aquí la variable se incluye como categórica, porque no es un mero control, sino que el interés radica precisamente en ver cómo determina la variable edad la probabilidad de formar una pareja endógama (cuando no podemos seleccionar a los inmigrantes que llegaron solos).

Como variables de control, todos los modelos incluyen la nacionalidad del cónyuge en el nacimiento y en el nivel de instrucción. Ambas variables se pueden utilizar sin demasiados problemas en este análisis transversal, puesto que la educación no debe haber variado sustancialmente entre el momento de la formación de la pareja y el momento de la encuesta, y la nacionalidad española, que sí podría haberlo hecho, se considera sólo cuando se tiene desde el nacimiento.

Los modelos no incluyen variables estructurales. Las variables estructurales, como tamaño del colectivo, segregación territorial o relación de masculinidad, deberían calcularse para cada colectivo individualmente. Los datos del Padrón Municipal permiten obtener estos indicadores, pero la Encuesta Nacional de Inmigrantes no permite realizar el análisis desagregado por colectivos (a excepción de Marruecos, Ecuador y Rumanía), debido al reducido número de casos.

\section{Niveles de endogamia de la población inmigrada. \\ Situación de pareja y edad en el momento de migrar}

El gráfico 2 muestra las proporciones de endogamia (uniones dentro del mismo grupo sobre el total de uniones de inmigrantes) por sexo y origen. Contrariamente a las proporciones de endogamia que suelen presentarse a partir de estocs de parejas ${ }^{7}$, estas están basadas exclusivamente en las parejas formadas por inmigrantes que llegaron sin pareja a España, con independencia de la edad de llegada. Los niveles de endogamia superan el $40 \%$ en todos los orígenes a excepción de las personas provenientes de la Europa de los Quince y, para las mujeres, las pertenecientes a la Europa de los Quince y las latinoamericanas. Aproximadamente el $80 \%$ de las parejas de africanos y africanas formadas tras la migración son endógamas. En el caso de los hombres, buena parte de estas

7. Es habitual calcular estas proporciones a partir de datos censales u otras fuentes transversales que no permiten conocer la situación de pareja en el momento de la migración. 
Gráfico 2. Proporciones de endogamia por sexo y origen de los emigrantes que llegaron solos (llegados después de 1991)

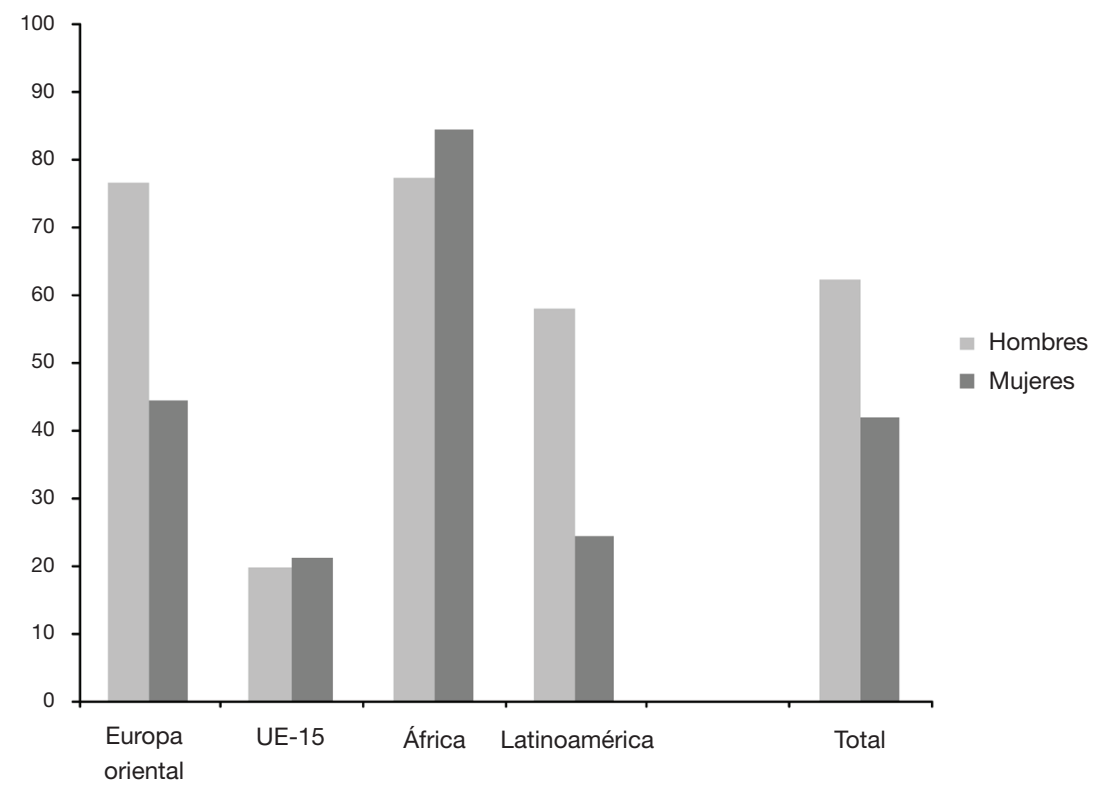

Fuente: Encuesta Nacional de Inmigrantes, INE, elaboración propia.

Nota: casos ponderados.

uniones, un 44\% (González Ferrer, 2011), se corresponden con matrimonios celebrados en el país de origen con cónyuges que no residían en España y que migraron posteriormente a este país.

Una cifra similar se observa para los hombres de la Europa Oriental (mayormente unidos con mujeres de su mismo origen). Los niveles de endogamia entre los latinoamericanos alcanzan el 60\%. En el otro extremo, los niveles de endogamia más bajos se observan entre la población procedente de la Europa de los Quince. Sólo uno de cada cinco inmigrantes de estos países se ha unido con un cónyuge del mismo país tras la migración. El resto lo ha hecho mayoritariamente con un cónyuge de origen español (resultados no presentados aquí). Después de los europeos, las mujeres de origen latinoamericano presentan los niveles de endogamia más bajos. Sólo el $25 \%$ se ha unido tras la migración con un cónyuge del mismo origen, el resto lo ha hecho con hombres de origen español. Lo mismo ocurre entre las mujeres de la Europa Oriental. En este caso, aproximadamente el $60 \%$ se ha unido con un cónyuge español.

Con el objetivo de comparar las diferencias observadas entre orígenes y sexos, se han diseñado modelos de regresión logística. El objetivo de los modelos no es establecer una relación de tipo causal entre las variables, sino más bien comparar las diferencias entre orígenes teniendo en cuenta un reducido grupo 
de variables a modo de estandarización. La tabla 2 informa de las variables, del número de casos y de los coeficientes obtenidos en la regresión, junto con su significancia estadística.

De acuerdo con el primer panel, que limita el universo de estudio a los que llegaron sin pareja, los hombres más endógamos son los de la Europa Oriental, seguidos de los africanos y los latinoamericanos. Los inmigrantes de la Europa de los Quince son los que tienen una probabilidad menor de ser endógamos. Entre las mujeres, la probabilidad de las africanas de formar una unión endógama es dos veces más alta que entre las de la Europa Oriental (valor tomado como referencia). A menor educación, mayor es la probabilidad de formar una pareja endógama, tanto para hombres como para mujeres. Los inmigrantes con menos de primaria son hasta seis veces más propensos a unirse con una persona de su mismo origen que los que tienen estudios universitarios. En lo que se refiere a la nacionalidad, aquellos nacidos en el extranjero que disponen de nacionalidad española desde el nacimiento son significativamente menos propensos a unirse con una persona de su mismo origen. La edad de llegada no parece tener un efecto demasiado relevante, especialmente para los hombres. Este resultado sugiere que, una vez controlado el efecto de la situación conyugal, la edad de llegada pierde valor por sí misma a la hora de determinar las opciones de emparejamiento de los inmigrantes.

El segundo panel reproduce el mismo modelo tomando como referencia al conjunto de los inmigrantes. El modelo añade, sin embargo, una variable que permite distinguir entre los que tenían pareja en el momento de migrar y los que no. El resultado es muy revelador. Los que migraron sin tener pareja tienen una probabilidad siete veces menor de estar unidos con alguien del mismo origen que los que tenían pareja en el momento de la llegada. Las diferencias entre unos y otros son incluso más acusadas entre las mujeres. El efecto de las variables de control, educación y nacionalidad española desde el nacimiento es parecido al del modelo anterior.

En el tercer panel, se muestran los resultados del modelo 3, que considera a todos los inmigrantes pero los clasifica por edad de llegada. En relación con esta variable, el modelo informa que la endogamia es más elevada entre aquellos inmigrantes que llegaron a edades más avanzadas, simplemente por el hecho de que entre estos hay una proporción mayor de inmigrantes en pareja en el momento de llegar (como se ha visto en el gráfico 1). En efecto, en el primer panel, donde sólo teníamos a los que llegaban solos, la edad de llegada pierde significación para los hombres y tiene un efecto muy ligero en las mujeres. Estos resultados muestran que la edad de llegada constituye un criterio apropiado para seleccionar un universo de inmigrantes con poco riesgo de haber formado pareja antes de la migración. Este tipo de filtro también se puede hacer en fuentes clásicas de tipo transversal, porque la información sobre el año de llegada suele estar disponible (Censo y EPA).

Los hombres que llegaron antes de los veinte años tienen una probabilidad menor de estar emparejados con mujeres del mismo país que la observada entre los que llegaron con treinta y cinco años o más. Entre los veinte y los veintinue- 
Tabla 2. Modelos logísticos multivariados de endogamia matrimonial (propensión a estar con un cónyuge del mismo país de nacimiento)

2.1. Universo: llegados solteros

País de nacimiento
Europa Oriental (ref.)
UE-15
África
Latinoamérica
Otros
Nacionalidad esp. de
Extranjero (ref.)
Español
Nivel educativo
Primaria incompleta
Primaria
Secundaria
Universidad (ref.)
Edad a la llegada
Constante
$\mathrm{N}$
-2 log likelihood

\begin{tabular}{|c|c|c|c|c|c|}
\hline \multicolumn{3}{|c|}{ Hombres } & \multicolumn{3}{|c|}{ Mujeres } \\
\hline$\%$ & $\operatorname{Exp}(\mathrm{B})$ & & $\%$ & $\operatorname{Exp}(B)$ & \\
\hline 18,1 & 1,00 & & 22,8 & & \\
\hline 16,6 & 0,04 & $* * *$ & 10,9 & 0,39 & $* * *$ \\
\hline 21,4 & 0,47 & $* * *$ & 8,7 & 2,04 & 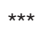 \\
\hline 40,1 & 0,41 & $\star * *$ & 55,4 & 0,61 & $* \star *$ \\
\hline 3,8 & 0,24 & $* * *$ & 2,3 & 1,11 & \\
\hline
\end{tabular}

2.2. Universo: llegados en cualquier situación de pareja (I)

\begin{tabular}{|c|c|c|c|c|c|c|}
\hline \multicolumn{7}{|c|}{ Hombres } \\
\hline \multirow{2}{*}{\multicolumn{7}{|c|}{ País de nacimiento (agrupado) }} \\
\hline & & & & & & \\
\hline Europa Oriental (ref.) & 22,3 & \multicolumn{2}{|l|}{1,00} & 24,4 & \multicolumn{2}{|l|}{1,00} \\
\hline UE-15 & 17,4 & 0,09 & 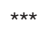 & 13,4 & 0,30 & *** \\
\hline África & 15,7 & 0,61 & ** & 9,6 & 1,43 & * \\
\hline Latinoamérica & 40,8 & 0,43 & 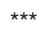 & 50,1 & 0,53 & *** \\
\hline Otros & 3,8 & 0,20 & 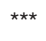 & 2,4 & 0,54 & * \\
\hline \multicolumn{7}{|c|}{ Nacionalidad española de nac. } \\
\hline Extranjero (ref.) & 92,1 & 1,00 & & 89,8 & 1,00 & \\
\hline Español & 7,9 & 0,57 & *** & 10,2 & 0,37 & *** \\
\hline \multicolumn{7}{|l|}{ Nivel educativo } \\
\hline Primaria incompleta & 2,4 & 5,72 & *** & 2,1 & 4,25 & *** \\
\hline Primaria & 17,1 & 3,55 & *** & 15,8 & 2,76 & *** \\
\hline Secundaria & 59,3 & 2,56 & 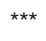 & 58,7 & 1,92 & *** \\
\hline Universidad & 21,1 & 1,00 & & 23,4 & 1,00 & \\
\hline \multicolumn{7}{|l|}{ Pareja a la llegada } \\
\hline Sin pareja & 34,9 & 0,15 & *** & 39,9 & 0,10 & *** \\
\hline Con pareja (ref.) & 65,1 & 1,00 & & 60,1 & 1,00 & \\
\hline Constante & & 9,08 & & & 4,76 & \\
\hline $\mathrm{N}$ & 3.038 & & & 3.738 & & \\
\hline 2 log likelihood & & $2.669,97$ & & & $3.732,18$ & \\
\hline
\end{tabular}

${ }^{* \star *} p<.001,{ }^{* \star} p<.01,{ }^{*} p<.05$

$88,7 \quad 1,00 \quad 85,1$

$11,3 \quad 0,64 \quad * \quad 14,9 \quad 0,43 \quad * * *$

$\begin{array}{rrrrrr}1,4 & 6,78 & * * & 1,4 & 6,73 & * * * \\ 11,8 & 4,61 & * \star * & 11,3 & 2,55 & * \star * \\ 62,5 & 2,85 & * \star * & 60,2 & 1,99 & * * * \\ 24,3 & 1,00 & * \star * & 27,0 & 1,00 & \\ 26,36 & 1,02 & & 26,1 & 0,94 & \text { *** } \\ & 0,74 & & & 1,70 & \end{array}$

$954 \quad 1.454$

$1.620,08$

Fuente: Elaboración propia a partir de los datos de la Encuesta Nacional de Inmigrantes 2007. 
Tabla 2. Modelos logísticos multivariados de endogamia matrimonial (propensión a estar con un cónyuge del mismo país de nacimiento) (continuación)

\begin{tabular}{|c|c|c|c|c|c|c|}
\hline \multicolumn{7}{|c|}{ 2.3. Universo: llegados en cualquier situación de pareja (II) } \\
\hline & \multicolumn{3}{|c|}{ Hombres } & \multicolumn{3}{|c|}{ Mujeres } \\
\hline & $\%$ & $\operatorname{Exp}(B)$ & & $\%$ & $\operatorname{Exp}(B)$ & \\
\hline \multicolumn{7}{|c|}{ País de nacimiento (agrupado) } \\
\hline Europa Oriental (ref.) & 22,3 & 1,00 & & 24,4 & 1,00 & \\
\hline UE-15 & 17,4 & 0,07 & *** & 13,4 & 0,39 & *** \\
\hline África & 15,7 & 0,38 & *** & 9,6 & 1,33 & \\
\hline Latinoamérica & 40,8 & 0,41 & *** & 50,1 & 0,53 & ** \\
\hline Otros & 3,8 & 0,19 & *** & 2,4 & 0,59 & * \\
\hline \multicolumn{7}{|c|}{ Nacionalidad española de nac. } \\
\hline Extranjero (ref.) & 92,1 & 1,00 & & 89,8 & 1,00 & \\
\hline Español & 7,9 & 0,47 & *** & 10,2 & 0,33 & $* * *$ \\
\hline \multicolumn{7}{|l|}{ Nivel educativo } \\
\hline Primaria incompleta & 2,4 & 7,28 & $* * *$ & 2,1 & 3,89 & *** \\
\hline Primaria & 17,2 & 4,64 & $* * *$ & 15,8 & 3,17 & *** \\
\hline Secundaria & 59,3 & 2,72 & *** & 58,7 & 1,89 & *** \\
\hline Universidad (ref.) & 21,1 & 1,00 & & 23,4 & 1,00 & \\
\hline \multicolumn{7}{|l|}{ Edad a la llegada } \\
\hline$<15$ & 0,6 & 0,06 & $* * *$ & 0,9 & 0,31 & $* * *$ \\
\hline $15-19$ & 4,0 & 0,11 & *** & 7,6 & 0,55 & *** \\
\hline $20-24$ & 18,6 & 0,31 & *** & 23,7 & 0,51 & *** \\
\hline $25-29$ & 24,5 & 0,30 & *** & 23,9 & 0,61 & $* * *$ \\
\hline $30-34$ & 18,8 & 0,50 & $* \star *$ & 17,1 & 0,65 & $* * *$ \\
\hline$>=35$ (ref.) & 33,5 & 1,00 & & 26,7 & 1,00 & \\
\hline Constante & & 9,49 & & & 2,45 & \\
\hline$N$ & 3.032 & & & 3.733 & & \\
\hline 2 log likelihood & & $2.865,43$ & & & $4.559,27$ & \\
\hline
\end{tabular}

Fuente: Elaboración propia a partir de los datos de la Encuesta Nacional de Inmigrantes 2007.

ve años, observamos niveles parecidos. Es a partir de los treinta años cuando la probabilidad de estar unidos con un cónyuge del mismo origen aumenta considerablemente. Entre las mujeres, observamos una pauta similar con algunas diferencias en relación con los hombres. La probabilidad de estar emparejado en el momento de la encuesta con un hombre del mismo país aumenta con la edad de llegada. Las mujeres que llegaron a edades más avanzadas es más probable que estén emparejadas con alguien del mismo origen. Los niveles de endogamia de las mujeres que llegaron a edades jóvenes son, en términos relativos, mucho más elevados que los de los hombres a las mismas edades.

\section{En resumen y conclusión}

En este trabajo, se han analizado los niveles de endogamia de la población inmigrada residente en España en 2007 con datos de la Encuesta Nacional de Inmigrantes, publicados por el Instituto Nacional de Estadística en 2008. 
La encuesta proporciona una información desconocida hasta el momento que resulta clave para analizar las pautas de emparejamiento de los inmigrantes: la situación conyugal de los inmigrantes en el momento de la llegada. Sobre la base de las parejas que los inmigrantes formaron después de migrar, se han calculado los niveles de endogamia de estos inmigrantes agrupados en cuatro grandes colectivos según procedan de África, América Latina, Europa de los Quince o Europa del Este. Los resultados muestran que los niveles de endogamia varían en función de la procedencia de los inmigrantes y, en algunos casos, entre hombres y mujeres de un mismo origen. Es posible que el comportamiento de estos grupos no sea representativo de todos los colectivos que los integran. La disponibilidad de datos no ha permitido este análisis. En resumen, los africanos muestran los niveles de endogamia más elevados. Aproximadamente cuatro de cada cinco inmigrantes africanos se han unido tras la migración con un cónyuge del mismo país. Estos resultados son coherentes con los hallazgos de otras investigaciones, que señalan, en el caso de los marroquíes, una fuerte tendencia a unirse dentro del mismo grupo incluso entre las segundas generaciones que no nacieron en Marruecos (Esteve y Jiménez, 2010). Las explicaciones a este comportamiento a menudo acuden a la religión y a la posición socioeconómica de estos colectivos.

Las explicaciones de tipo sociocultural son menos relevantes para explicar las diferencias que se observan por sexo dentro de un mismo colectivo. Es el caso paradigmático de los inmigrantes de la Europa Oriental y de América Latina. En ambos casos, los hombres forman pareja mayoritariamente con mujeres de su mismo país, mientras que más de la mitad de las mujeres se emparejan con hombres nacidos en España. Algunos autores han apuntado la importancia de las características estructurales como posibles determinantes de este comportamiento desigual entre hombres y mujeres. En sus inicios, la migración de América Latina y la Europa Oriental fue muy feminizada. Esto podría explicar la mayor propensión de las mujeres a formar pareja con hombres españoles. Sin embargo, hay elementos para pensar que puede haber razones del mercado matrimonial español que justifiquen esta asimetría por sexo. Futuras investigaciones deberán abordar el papel que la dinámica interna del mercado matrimonial español haya podido tener en estos matrimonios.

Finalmente, la baja endogamia entre los inmigrantes de la Europa de los Quince prueba la relativa facilidad con la que se establecen uniones con la población nacida en España. Algunos autores vinculan este hecho con la construcción de un espacio europeo en el que se han reducido las barreras a la movilidad profesional y residencial, especialmente entre las generaciones más recientes. En este sentido, si restringimos la observación a los europeos que llegaron a España sin pareja, nos encontramos efectivamente con una población que migró mayoritariamente durante su etapa formativa, con el apoyo de los crecientes programas de intercambio estudiantil como el Erasmus, en una edad y en unas circunstancias que directamente les enmarcaban en el mercado matrimonial español (King y Ruiz-Gelices, 2003). 
El segundo objetivo del trabajo era examinar posibles alternativas para identificar a los inmigrantes que formaron pareja en España cuando la información sobre la situación de pareja en el momento de la llegada no era conocida. Los resultados muestran claramente que, cuando se considera a todos los inmigrantes, los niveles de endogamia de todas las uniones son muy superiores a las de las uniones que se formaron tras la migración. La justificación de esta diferencia es evidente. En el primer caso, se están incluyendo parejas formadas antes de la migración que, en la práctica totalidad de los casos, son entre cónyuges del mismo país. A medida que aumenta la edad del inmigrante, la probabilidad de estar emparejado en el momento de la migración también aumenta. Entre las mujeres, esta proporción aumenta a una edad más temprana, debido, entre otros aspectos, al calendario más temprano de entrada a la unión.

Cuando la situación de pareja a la llegada no se conoce, los resultados sugieren que la edad de llegada es un buen filtro para seleccionar a aquellos inmigrantes que formaron pareja tras la migración. La probabilidad de tener pareja en el momento de la migración aumenta con la edad a la llegada. Así, seleccionando a los inmigrantes que llegaron antes de los dieciséis años, se garantiza prácticamente que la mayoría de parejas que formaron estos inmigrantes fueron posteriores a la migración. Sin embargo, este es un criterio bastante restrictivo y que en algunos colectivos puede mermar considerablemente el número de casos. Los resultados sugieren que no es necesario usar el mismo umbral de edad para hombres y mujeres ni en función del colectivo. En el caso de los inmigrantes procedentes de la Europa de los Quince, la proporción de emparejado antes de los veinticinco años es muy reducida. En el caso de las mujeres africanas, sin embargo, la proporción de mujeres que tenía pareja a los veinte años era del $50 \%$. En cualquier caso, los resultados de este análisis no ofrecen una solución perfecta ni tampoco una edad ideal para filtrar a los inmigrantes. En función de los objetivos de cada investigación, deberán aplicarse los filtros que más convengan.

El principal problema asociado al uso de la edad de llegada como filtro es que sólo permite controlar en buena medida la dimensión temporal de la secuencia entre migración y formación de pareja, pero no tanto su dimensión espacial, como solicita el verso de la canción que da título a este artículo. Podemos descartar con más o menos fiabilidad aquellos inmigrantes que estaban unidos antes de venir, pero resulta difícil distinguir, entre los que lo hayan hecho con posterioridad, a aquellos que formaron pareja en España o en el extranjero. El lugar en el que se forma la pareja es altamente determinante del origen del esposo y, por lo tanto, de los niveles de endogamia. La literatura internacional ha destacado la importancia del fenómeno de la importación o esponsorización de cónyuges para referirse a los matrimonios entre inmigrantes ya residentes en destino con esposos o esposas que se encuentran en el país de origen (Lievens, 1999; González Ferrer, 2006, 2011). En el caso español, esta práctica no es común entre los inmigrantes de todos los orígenes, sino que es más frecuente entre los africanos y los marroquíes especialmente, de modo 
que el sesgo que se podría introducir con el filtro por edad afectaría de manera distinta a cada colectivo.

La próxima publicación de los datos del censo de 2011 abrirá nuevas oportunidades para la investigación en este ámbito. El número de casos permitirá muy probablemente realizar análisis específicos para los colectivos más representados, aunque se desconocerá la situación de pareja de los inmigrantes en el momento de llegada. Como ya lo fuera en los análisis realizados con el censo de 2001, la edad de llegada resultará una variable clave para seleccionar aquellos inmigrantes que formaron pareja tras la migración con mayor probabilidad. Será especialmente interesante poder comparar, utilizando la misma metodología, las transformaciones experimentadas por los niveles de endogamia de la población inmigrante en España transcurridos diez años clave en la evolución de los flujos migratorios. Si, en 2001, el contexto era de una población inmigrante creciente y mayoritariamente recién llegada, en 2011, el censo reflejará las características y las pautas matrimoniales de colectivos ya más asentados y que han dejado de crecer. ¿Qué implicaciones podrá tener este cambio coyuntural en los proyectos migratorios y familiares de los inmigrantes internacionales en España?

\section{Referencias bibliográficas}

Alba, R.D. y NeE, V. (1997). «Rethinking Assimilation Theory for a New Era of Immigration». International Migration Review, 4, 826-874.

BLACKWELL, D.L. (1998). «Marital homogamy in the United States: The influence of individual and parental education». Social Science Research, 27, 159-188.

Blau, P.M.; Blum, T.C. y SCHWARTZ, J.E. (1982). «Heterogeneity and intermarriage». American Sociological Review, 47 (1), 45-62.

Bledsoe, C.H.; René, H. y PAPA, S. (2007). «High fertility Gambians in low fertility Spain: The dynamics of child accumulation across transnational space». Demographic Research, 16-12, 375-412.

Collet, B. y Philipppe, C. (dir.) (2008). Mixité (s). Variations autour d'une notion transversale. París: L'Harmattan.

Cortina, C.; Esteve, A. y CABRÉ, A. (2009). "Con quién se unen los latinoamericanos en España?: Respuestas a partir de tres fuentes estadísticas». Notas de Población, 86, 19-38.

Cortina, C.; Esteve, A. y Domingo, A. (2006). "Crecimiento y singularidades demográficas de los matrimonios de extranjeros en España». Migraciones, 20, $75-105$.

- (2008). «Marriage patterns of foreign born population in a new country of immigration: the case of Spain». International Migration Review, 42 (4), 877-902.

Cortina, C.; GARCia, T. y Esteve, A. (2009). «Migración, ocupación y matrimonio: Una aproximación a las relaciones de género de las parejas mixtas en España». Estudios Demográficos y Urbanos, 71, 24 (2), 293-321.

DEVOLDER, D. y TREVINO, R. (2007). «Efectos de la inmigración extranjera sobre la evolución de la natalidad y de la fecundidad en España». Papers de Demografia, 321.

EsteVE, A. y Bueno, X. (2010). «Tras el rastro estadístico de las parejas de inmigrantes en España». Revista de Estadística Española, 52 (173), 91-125. 
Esteve, A. y JiméneZ, E. (2010). «La formación de la pareja entre marroquíes en España». Migraciones, 27, 83-110.

FILHON, A. y VARRO, G. (2005). "Les couples mixtes, une catégorie hétérogène». En: LefËvre, C. y Filhon, A. (eds.). Histoires de familles, histoires familiales, 483-501.

GonZÁlez Ferrer, A. (2006). «Who do immigrants marry?: Partner’s choice among single immigrants in Germany». European Sociological Review, 22 (2), 171-185.

- (2008). Estrategias familiares y laborales en la emigración: Reagrupación familiar, elección de parejas y empleo de los inmigrantes en el país de destino. Madrid: Consejo Económico y Social.

- (2011). «Explaining the labour performance of immigrant women in Spain: The interplay between family, migration and legal trajectories». International Journal of Comparative Sociology, 52 (1-2), 63-78.

Gordon, M. (1964) Assimilation in American Life. Oxford, Oxford University Press.

JaCOBS, J.A. y LABOV, T.G. (2002). "Gender Differentials in Intermarriage Among Sixteen Race and Ethnic Groups». Sociological Forum, 17 (4), 621-646.

Kalmijn, M. (1998). "Intermarriage and Homogamy: Causes, Patterns, Trends». Annual Review of Sociology, 24, 395-421.

KinG, R. y Ruiz-Gelices, E. (2003). «International student migration and the European "Year Abroad": Effects on European identity and subsequent migration behaviour». International Journal of Population Geography, 9 (3), 229-252.

LÁZARO GONZÁlEZ, I. (2002). «La reagrupación familiar de los extranjeros en España: Previsible incidencia en la futura directiva sobre el derecho a la reagrupación familiar». Migraciones, 12, 43-79.

LiEvens, J. (1999) «Family-Forming Migration from Turkey and Morocco to Belgium: The Demand of Marriage Partners from the countries of origin». International Migration Review, 33 (3), 717-744.

Massey, D. (1985). «Ethnic Residential Segregation: A Theoretical Synthesis and Empirical Review». Sociology and Social Research, 69, 315-350.

MCCAA, R. (1993). "Ethnic intermarriage and gender in New York City». Journal of Interdisciplinary History, 24 (2), 207-231.

MERTON, R.K. (1941). «Intermarriage and the Social Structure: Fact and Theory». Psychiatry, 4, 361-374.

PAGNINI, D.L. y Morgan, S.P. (1990). «Intermarriage and social distance among U.S. immigrants at he turn of the century». American Sociological Review, 96, 405-432.

Portes, A. y Zhou, M. (1993). «The New Second Generation: Segmented Assimilation and Its Variants among Post-1965 Immigrant Youth». Annals of the American Academy of Political Sciences, 53, 75-98.

PRICE, C.A. y ZubrZYCKI, J. (1962). "The use of inter-marriage statistics as an index of assimilation». Population Studies, 16, 58-69.

QIAN, Z. (1998). "Changes in Assortative Mating. The Impact of Age and Education, 1970,1990». Demography, 35 (3), 279-292.

Reher, D. y Requena, M. (2009). "The National Immigrant Survey of Spain: A new data source for migration studies in Europe». Demographic Research, 20, 253-278.

RodrígueZ, D. (2004). «Inmigración y mestizaje hoy: Formación de matrimonios y familias transnacionales de inmigrantes en Cataluña». Migraciones, 16, 77-120.

- (2006). «Mixed Marriages and Transnational Families in the Intercultural Context: A Case Study of African-Spanish Couples in Catalonia». Journal of Ethnic and Migration Studies, 32 (3), 403-433. 
Rodríguez Marcos, E. (2006). Familias interculturales: La construcción de la interculturalidad de lo micro social a lo macro social. Publicaciones de la Universidad Pontificia de Salamanca.

Roig, M. y Castro Martín, T. (2007). «Childbearing patterns of foreign women in a new immigration country: The case of Spain». Population English Edition, 62 (3), 351-380; Population Édition Française, 62 (3), 419-446.

SовоткA, T. (2008). «The rising importance of migrants for childbearing in Europe». Demographic Research, 19 (9), 225-248. 